\title{
Satisfação e percepção do usuário do SUS sobre o serviço público de saúde
}

I ${ }^{1}$ Suzely Adas Saliba Moimaz, ${ }^{2}$ Jeidson Antônio Morais Marques, ${ }^{3}$ Orlando Saliba, ${ }^{4}$ Cléa Adas Saliba Garbin, ${ }^{5}$ Lívia Guimarães Zina, ${ }^{6}$ Nemre Adas Saliba I

Resumo: A avaliação do grau de satisfação dos usuários do sistema de saúde é um importante indicador a ser considerado no planejamento das açôes. O objetivo deste estudo foi avaliar o grau de satisfação de usuários dos serviços de saúde pública municipal quanto aos serviços utilizados. Este estudo tipo inquérito foi conduzido em cinco municípios do Estado de São Paulo. A amostra foi selecionada de forma estratificada e aleatória, sendo entrevistados 471 chefes de família ou respectivos cônjuges. Os dados qualitativos foram analisados pelo método de Análise de Conteúdo, e os dados quantitativos foram processados utilizando-se o software estatístico Epi Info. Dentre os usuários dos cinco municípios, 93,0\% utilizam o serviço municipal de saúde. Para 72,0\%, os serviços de saúde prestados estão resolvendo os problemas e necessidades da população. Com base no acesso ao atendimento, 57,6\% da população queixaram-se da presença de filas para o atendimento. Mais da metade (69,5\%) afirmou ter confiança na equipe de saúde; no entanto, muitos relatos demonstraram a carência de um atendimento humanizado. Em relação aos serviços de saúde, 61,7\% classificaram-os como ótimo ou bom. Conclui-se que a maior parte dos usuários mostrou-se satisfeita com os serviços de saúde municipais, apesar da grande quantidade de queixas quanto ao atendimento, falta de humanização e acolhimento, deficiência de recursos físicos e materiais. A percepção do usuário é de extrema importância ao se dimensionar o reflexo das ações que vem sendo desenvolvidas no setor saúde, e serve como vetor de direcionamento e planejamento do serviço.

> Palavras-chave: Avaliação em saúde, Programa Saúde da Família, Satisfação dos Consumidores, Serviços de Saúde.
1 PhD, Professora Adjunta, Programa de Pós-graduação em Odontologia Preventiva e Social - Faculdade de Odontologia de Araçatuba - UNESP. Endereço eletrônico: sasaliba@foa. unesp.br.

2 PhD, Aluno(a) de pósgraduação, Programa de Pós-graduação em Odontologia Preventiva e Social - Faculdade de Odontologia de Araçatuba - UNESP.

${ }^{3} \mathrm{PhD}$, Professor(a) Titular, Programa de Pós-graduação em Odontologia Preventiva e Social - Faculdade de Odontologia de Araçatuba - UNESP.

${ }^{4}$ PhD, Professora Adjunta, Programa de Pós-graduação em Odontologia Preventiva e Social - Faculdade de Odontologia de Araçatuba - UNESP.

${ }^{5} \mathrm{PhD}$, Aluno(a) de pósgraduação, Programa de Pós-graduação em Odontologia Preventiva e Social - Faculdade de Odontologia de Araçatuba - UNESP.

${ }^{6} \mathrm{PhD}$, Professor(a) Titular, Programa de Pós-graduação em Odontologia Preventiva e Social - Faculdade de Odontologia de Araçatuba - UNESP.

Recebido em: 15/12/2009. Aprovado em: 05/05/2010. 
O Sistema Único de Saúde (SUS) surgiu no Brasil a partir de um histórico de lutas do movimento sanitário brasileiro com a finalidade de facilitar o atendimento de saúde (Roncalli, 2003). Na Constituição Federal de 1988, ficou explicitada a criação de um sistema de saúde estruturado, tendo como base a descentralização e o fortalecimento do poder municipal, além de estabelecer a participação da sociedade organizada na administração do setor saúde e o controle social por meio dos Conselhos e Conferências Municipais de Saúde, visando a formulação, implantação, controle e avaliação das políticas de saúde (Brasil, 1988).

Em 1990, foram publicadas as Leis $\mathrm{n}^{\mathrm{o}} \mathrm{s} 8.080 / 90$ e 8.142/90, que institucionalizaram e regulamentaram a participação popular e o controle social na gestão da saúde, tendo como instâncias legalmente instituídas as Conferências e os Conselhos de Saúde. Estes são descritos como órgãos permanentes, deliberativos e normativos do SUS no âmbito municipal, que tem por competência formular estratégias e controlar a execução da política de saúde do município, inclusive nos seus aspectos econômicos e financeiros (BRASIL, 2006). As diretrizes do novo sistema de saúde serviram de base para estruturar as mudanças necessárias no sistema de saúde, favorecendo a descentralização das ações, de forma que cada município passasse a gerir as ações em saúde conforme as necessidades locais.

Visando à mudança do modelo hegemônico centrado na doença, em 1994 o Ministério da Saúde, a fim de consolidar o SUS, propôs aos municípios o Programa de Saúde da Família, posteriormente denominado Estratégia Saúde da Família (ESF), com o propósito de reorganizar a prática de atenção à saúde em novas bases, substituindo o modelo tradicional (MULLER et al., 2003). O documento que define as bases do programa destaca que, ao contrário do modelo tradicional, centrado na doença e no hospital, a ESF prioriza as ações de proteção e promoção à saúde dos indivíduos e da família, tanto adultos quanto crianças, sadios ou doentes, de forma integral e contínua (TRAD; BASTOS, 1998).

A ESF incorpora e reafirma os princípios básicos do SUS - universalização, descentralização, integralidade e participação da comunidade - e está estruturada a partir da Unidade Básica de Saúde da Família, na qual atuam profissionais de diversas áreas e formaçôes distintas. No entanto, apesar dos incentivos governamentais para a consolidação desta estratégia, observa-se que em muitos 
dos municípios nos quais a ESF foi implantada existem ainda grandes deficiências na execução dos trabalhos, com uma carência de maiores discussões sobre o modo de relação entre os profissionais e a população local (SENNA, 2002). Nestes casos, a ESF significou mais uma modificação institucional do que uma maior aproximação com o cotidiano das famílias.

Uma das especificidades que chama a atenção na proposta da ESF e nas próprias diretrizes do SUS diz respeito à participação da comunidade. O controle social é a expressão mais viva da participação da sociedade nas decisões tomadas pelo Estado no interesse geral, e suas manifestações mais importantes são o cidadão e o usuário no centro do processo de avaliação (CREVELIM, 2005). O controle social fortalecido com o advento do SUS e o incentivo à participação da comunidade pressupõem uma concepção do usuário com competência para avaliar e intervir modificando o próprio sistema (TRAD; BASTOS, 1998). Sendo a população em sua totalidade beneficiada com projetos e ações em saúde, a opinião dos usuários quanto à qualidade da atenção é considerada de extrema relevância quando se busca avaliar esses serviços. Assim, não se pode deixar de considerar sua percepção, a fim de contribuir para a melhoria do sistema de saúde (SANTOS, 1995; JESUS; CARVALHO, 2002). Importante também é saber como a população adscrita às equipes da ESF avalia o atendimento oferecido, de modo a repensar as práticas profissionais ou intervir sobre a forma de organização dos serviços, visando seu aprimoramento (RAMOS; LIMA, 2003). A participação livre e crítica dos usuários pode contribuir para o empoderamento / libertação deste grupo populacional, questôes estas essenciais para a viabilização de políticas de promoção da saúde e prevenção de agravos e controle de enfermidades (COTTA et al., 2008). Desse modo, a melhoria dos programas de saúde pode ser baseada no processo de avaliação apoiado na análise do sistema por meio da ótica do usuário, servindo de parâmetro para as ações e políticas de saúde.

Diversos estudos têm sido realizados, buscando avaliar o grau de satisfação de usuários dos serviços de saúde (SANTOS, 1995; KLOETZEL et al., 1998; SHIDER; PALM, 2002; MULLER, 2003; MATEOS et al., 2009). Um dos principais pontos discutidos nesse caminho é a crescente e cada vez mais consensual opinião de que a maioria das ações de saúde não tem levado em consideração a concordância e a aceitação do paciente (RESENDE et al., 
1999). Desse modo, foi objetivo deste trabalho avaliar o grau de satisfação dos usuários dos serviços de saúde pública municipal quanto aos serviços utilizados em cinco municípios da região noroeste do Estado de São Paulo, permitindo assim compreender a percepção do usuário e analisar a qualidade e eficiência dos serviços prestados pelo sistema de saúde local.

\section{Materiais e métodos}

Este estudoㄹ , tipo inquérito, fez parte do projeto de intervenção "Saúde Bucal da população: desenvolvimento de metodologia para a implementação da atenção odontológica no PSF", desenvolvido em 2003-2006, com a participação de docentes, alunos de pós-graduação, acadêmicos e técnicos da área da saúde, lideranças das comunidades, prefeitos, secretários municipais de Saúde, secretários municipais de Educação, membros dos Conselhos Municipais de Saúde e demais servidores do setor saúde dos municípios envolvidos.

O projeto buscou realizar o diagnóstico situacional de municípios do DRS-II (Departamento Regional de Saúde) e a análise dos modelos de gestão, criando ferramentas que estão auxiliando os serviços de saúde na sua reestruturação, além de capacitar os profissionais da Estratégia Saúde da Família - Equipe de Saúde da Família e Equipe de Saúde Bucal - capacitar os Conselheiros Municipais de Saúde e auxiliar na dinamização dos Conselhos Municipais de Saúde. Por fim, o projeto avaliou o grau de satisfação, conhecimento e percepção do usuário dos serviços de saúde pública municipal quanto aos serviços utilizados, sendo realizado trabalho educativo, domiciliar, com o intuito de transferir conhecimentos e conscientizar os membros das famílias moradoras dos municípios participantes a respeito da ESF e da dinâmica de funcionamento do serviço de saúde local, bem como do direito à participação no processo decisório das políticas públicas de saúde, e distribuído o Manual do Usuário pelos agentes comunitários de saúde (ACS), formulado para esse fim.

Este artigo apresenta os resultados referentes ao grau de satisfação do usuário. Dados referentes às demais vertentes do projeto foram apresentados em publicações prévicas (SALIBA et al., 2007; SANTOS et al., 2008). O estudo foi conduzido em cinco municípios de pequeno porte (Bilac, Clementina, Piacatu, Santópolis do Aguapeí e Gabriel Monteiro), localizados na região noroeste do Estado de São Paulo e com condiçōes demográficas e socioeconômicas semelhantes (IBGE, 2007). 
Quanto ao procedimento metodológico, o estudo caracterizou-se por ser uma pesquisa de campo, atuando o pesquisador no meio onde se desenrola a existência do fenômeno, tendo como técnica a entrevista, instrumento este que permite uma análise complexa do problema (RICHARDSON, 1999). Em um primeiro momento, foi feito o contato com a Prefeitura e Secretaria Municipal de Saúde de cada município, com o intuito de obter autorização para a realização da pesquisa, esclarecendo as autoridades responsáveis sobre a natureza e modo de condução da mesma, além de oficinas para discussão e formalização de parcerias.

Todos os municípios estudados têm implementada em seu sistema de saúde a Estratégia Saúde da Família. Para a avaliação do grau de satisfação dos usuários da rede pública de saúde, foram realizadas visitas domiciliares durante quatro meses. A amostra foi selecionada de forma estratificada e aleatória, por meio de sorteio das quadras nos bairros residenciais, sendo composta de 95 (Bilac), 95 (Clementina), 95 (Piacatu), 93 (Santópolis do Aguapeí) e 93 (Gabriel Monteiro) famílias. Foram entrevistados os chefes de cada família ou respectivos cônjuges pertencentes à amostra. Este número foi obtido a partir do cálculo da amostra para levantamentos epidemiológicos em saúde (WHO, 1997), baseado no número total de habitantes do município, considerando uma margem de erro igual a $10 \%$, sendo suficiente para que se faça inferência estatística dos dados.

Dentro da metodologia proposta, optou-se pela entrevista dirigida, realizada por pesquisadores previamente calibrados, utilizando-se para a coleta das informações um formulário semiestruturado, com 21 questôes abertas e fechadas, incluindo dados de identificação socioeconômica e educacional, utilização dos serviços locais de saúde, acesso às consultas, percepção e satisfação em relação ao atendimento prestado nas unidades de saúde e hospitais municipais - incluindo a odontologia - à equipe e a ESF e comentários sobre o sistema de saúde nos municípios. A distribuição da amostra segundo a classe social foi feita de acordo com o critério de classificação econômica proposto pela Associação Brasileira de Empresas de Pesquisa (ABEP, 2000). De acordo com a ABEP, a população brasileira está distribuída entre sete classes sociais: $\mathrm{A} 1$, correspondendo à renda familia média de $\mathrm{R} \$ 7.793,00$; A2, renda familiar média de $\mathrm{R} \$ 4.648,00 ; \mathrm{B}$, renda familiar média de $\mathrm{R} \$ 2.804,00$; $\mathrm{B} 2$, renda familiar média de $\mathrm{R} \$ 1.669,00$; C, renda familiar média de $\mathrm{R} \$ 927,00$; $\mathrm{D}$, renda familiar média de $\mathrm{R} \$ 424,00$, e classe E, com renda familiar média de R \$207,00. 
Cada chefe de família foi abordado em sua residência, durante o horário comercial (8-17 horas) e convidado a participar do estudo. A entrevista foi anotada e teve duração de dez minutos em média, sendo obtido o termo de consentimento livre esclarecido dos participantes da pesquisa. Foi realizado estudo piloto para validação e adequação do instrumento de coleta de dados.

Os dados qualitativos foram transcritos e analisados pelo método de Análise de Conteúdo (BARDIN, 2004). Dentre as técnicas de Análise de Conteúdo, foi realizada, neste trabalho, a análise categorial, funcionando através de operações de desmembramento do texto em unidades e em categorias segundo reagrupamentos analógicos. Os resultados foram então descritos conforme a distribuição nas categorias e apresentadas as falas dos sujeitos. Os dados quantitativos foram codificados, duplamente conferidos, digitados e processados utilizando o software estatístico Epi Info versão 3.2.2. Posteriormente, foi realizada a distribuição das frequências.

Este estudo se enquadra na modalidade de pesquisa de risco mínimo, de acordo com a Resolução no 196/96 do Conselho Nacional de Saúde, relativa a pesquisa em seres humanos, e teve a aprovação do Comitê de Ética e Pesquisa em Seres Humanos da Faculdade de Odontologia de Araçatuba - UNESP (processo número 2005-02187).

\section{Resultados e discussão}

A tabela 1 apresenta as características demográficas, socioeconômicas e de assistência à saúde dos sujeitos da pesquisa. No total, foram entrevistados 471 sujeitos, com idade entre 16 e 96 anos, média de 48 anos. Destes, quase $80 \%$ eram do sexo feminino, representados em sua maioria por donas de casa. Os maridos, na maior parte das vezes, estavam ausentes durante o horário das entrevistas. A amostra apresentou nível médio de escolaridade, já que mais da metade dos entrevistados havia completado o ensino fundamental. Apenas 8,9\% estudavam no momento da entrevista, sendo que metade destes em escola pública. Verificouse maior prevalência de distribuição da amostra entre classes sociais mais baixas, quando comparada à média nacional (ABEP, 2000). 
Tabela 1. Características demográficas, socioeconômicas e de assistência dos usuários do SUS. Brasill, 2009

\begin{tabular}{|c|c|c|}
\hline Características & $\mathbf{N}^{\dagger}$ & $\%$ \\
\hline \multicolumn{3}{|l|}{ Gênero } \\
\hline Feminino & 368 & 78,1 \\
\hline Masculino & 103 & 21,9 \\
\hline \multicolumn{3}{|l|}{ Escolaridade } \\
\hline Não estudaram & 79 & 16,9 \\
\hline Até ensino fundamental & 274 & 58,4 \\
\hline Até ensino médio & 86 & 18,3 \\
\hline Até ensino superior & 30 & 6,4 \\
\hline \multicolumn{3}{|l|}{ Classe social $\left(\mathrm{ABEP}^{\dagger}\right)$} \\
\hline Classe B1 & 7 & 1,5 \\
\hline Classe B2 & 15 & 3,2 \\
\hline Classe C & 166 & 35,3 \\
\hline Classe D & 267 & 56,8 \\
\hline Classe E & 15 & 3,2 \\
\hline \multicolumn{3}{|l|}{ Moradia } \\
\hline Própria & 379 & 80,5 \\
\hline Alugada & 68 & 14,4 \\
\hline Cedida & 22 & 4,7 \\
\hline Outros & 2 & 0,4 \\
\hline \multicolumn{3}{|c|}{ Utiliza o serviço de saúde público } \\
\hline $\operatorname{Sim}$ & 436 & 92,8 \\
\hline Não & 34 & 7,2 \\
\hline \multicolumn{3}{|c|}{ Já precisou de encaminhamento de saúde } \\
\hline Sim & 294 & 66,8 \\
\hline Não & 146 & 33,2 \\
\hline \multicolumn{3}{|c|}{ Resolubilidade - Os serviços estão resolvendo o problema da população } \\
\hline Sim & 315 & 72,1 \\
\hline Não & 122 & 27,9 \\
\hline
\end{tabular}

†Soma pode ser inferior a 471, pois alguns sujeitos não responderam todas as questôes. 
Quase a totalidade da amostra (93\%) relatou utilizar o SUS local em situações diversas, como consultas de rotina, atendimento fisioterápico e odontológico, cirurgias, emergências e internação (tabela 1). Em geral, tanto o entrevistado quanto seus familiares costumam frequentar a unidade de saúde da família pelo menos uma vez por mês. Sessenta e sete por cento já precisaram de encaminhamento para especialidades médicas; destes, $83 \%$ concluiram o tratamento com sucesso.

Para $72 \%$, os serviços de saúde prestados estão resolvendo os problemas e necessidades da população (tabela 1). Para aqueles que discordaram, as razôes alegadas para a não-resolubilidade do sistema foram a falta de remédios, poucas vagas e demora para atendimento, dificuldade no sistema de referência e contrarreferência e poucos profissionais em atividade.

Com base no acesso ao atendimento, $57,6 \%$ da população responderam que existe fila para o atendimento e 54,5\% afirmaram que existe uma demora excessiva entre o dia de agendamento da consulta e/ou exame até o dia do atendimento. Este dado está relacionado à percepção pessoal acerca desse tempo de espera, variando de um dia a um ano ou mais. A ordem do atendimento dos serviços de saúde foi citada como ordem de chegada (82,9\%), agendamento $(9,1 \%)$ ou outros $(8 \%)$. De modo geral, não houve planejamento de saúde nos municípios avaliados, tomando-se como ponto de partida a realidade local, com relação aos problemas de saúde, os recursos humanos, físicos e o apoio da comunidade. Groisman et al. (2005) falam em seu estudo da importância de tal visão num processo de construção de um modelo de atenção em saúde segundo os princípios do SUS.

A insatisfação quanto ao tempo de espera por um tratamento tem-se mostrada rotineira nos servicos, como foi relatado por Andrade e Ferreira (2006), em estudo conduzido no município de Pompéu (MG). Em outra pesquisa (FRANCO; CAMPOS, 1998), realizada em um ambulatório de pediatria do SUS, a demora para receber atendimento foi o principal problema apontado pelos usuários. A espera prolongada foi também identificada (KLOETZEL et al., 1998) como o motivo número um de insatisfação da clientela, além da reclamação de haver insuficiência quanto à possibilidade de agendamento de novas consultas. $\mathrm{O}$ 
atendimento dos pacientes por meio da livre demanda é uma prática recorrente nos serviços públicos nacionais e remonta a uma tradição curativista. Percebe-se que, apesar da importância desse tipo de organização da demanda, a prática do agendamento do usuário deve ser utilizada concomitantemente, já que auxilia na otimização do serviço, diminui o número de pacientes na espera e evita as filas nas unidades de saúde.

Quanto aos critérios de humanização no atendimento à saúde, 69,5\% confiam em todos os profissionais de saúde da rede pública pelos quais foram atendidos. Mais da metade $(63,7 \%)$ já havia recebido informação sobre prevenção; para $72,9 \%$, os profissionais de saúde que os atenderam explicaram sobre a doença que tinham ou têm, e os procedimentos que foram realizados para o tratamento (70,3\%); 92\% já receberam prescrição de medicamentos no SUS, e para 83,1\% destes os profissionais explicaram a respeito da administração do remédio; $73 \%$ relataram que receberam atenção adequada às suas queixas por parte dos profissionais.

Ficou claro que o desempenho profissional é avaliado pelo usuário, através do interesse demonstrado no exame físico, nas perguntas feitas e orientações dadas, bem como na resolutividade das condutas. O bom atendimento, baseado na escuta do usuário, e o bom desempenho profissional propiciam o vínculo do binômio usuário-serviço de saúde. Esse vínculo otimiza o processo da assistência, permitindo que os profissionais conheçam seus pacientes e as prioridades de cada um, facilitando-lhes o acesso. Ficou evidenciada, nas falas dos sujeitos desta pesquisa, uma preocupação com a humanização do atendimento, bem como com uma forma de organização que leve em conta a escuta de suas necessidades como usuário.

Sobre os serviços de saúde geral dos municípios avaliados, $61,7 \%$ os classificaram como ótimo e bom (gráfico 1). Apesar das queixas relatadas, o usuário mostrou-se satisfeito com os serviços públicos de saúde oferecidos. Guedes e Garcia (2001) estudaram a satisfação do usuário do serviço e verificaram que as respostas dos usuários acerca de seu nível de satisfação quanto ao atendimento nos serviços do SUS apresentaram um direcionamento para respostas positivas (ficando em torno de $40 \%$ a $50 \%$ ), pois há sempre um receio de que as respostas 
1428 desfavoráveis possam resultar em algum tipo de sanção no atendimento. Por outro lado, quando o entrevistador não é uma pessoa da comunidade, pode acontecer o oposto e ocorrer uma tendência para os pacientes relatarem mais respostas negativas. Tal situação foi observada pela equipe deste projeto, ao notar o constrangimento inicial dos entrevistados ao serem abordados sobre o tema, e o alívio e desenvoltura destes ao descreverem com maior ênfase suas percepções, logo após serem esclarecidos sobre os propósitos do estudo.

\section{Gráfico 1. Avaliação dos serviços de saúde pelos usuários. Brasill, 2009.}

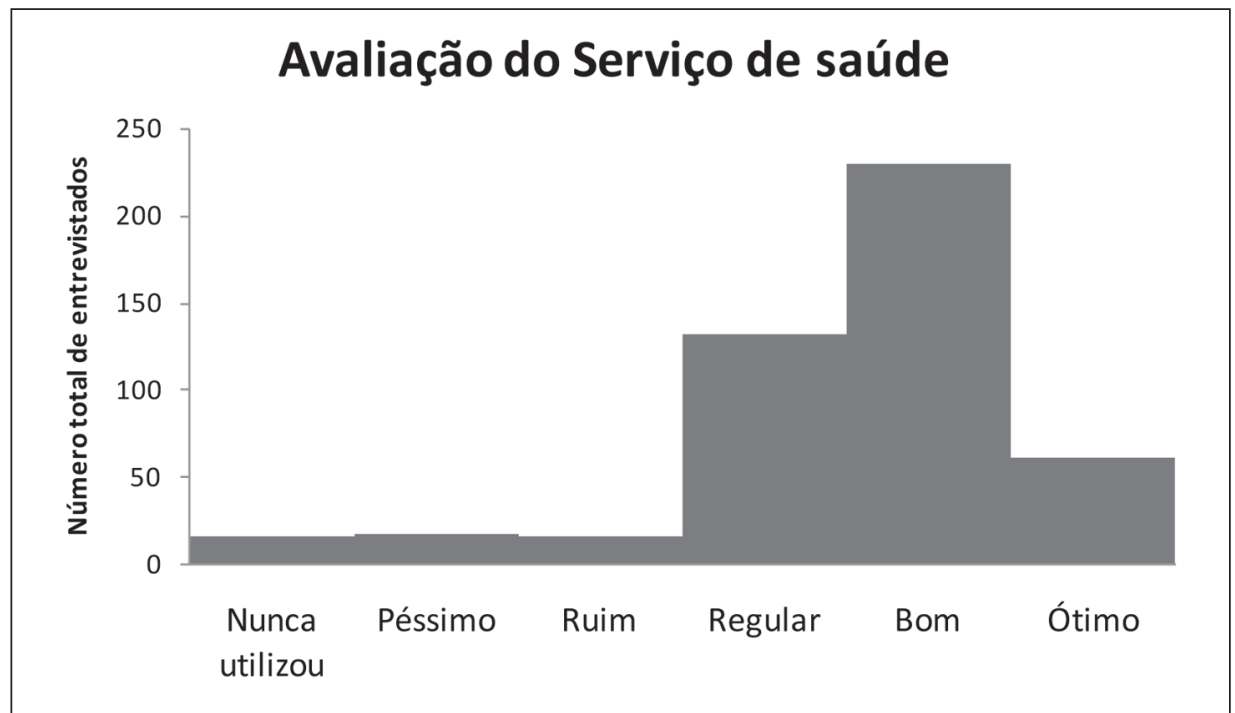

Ao avaliar os serviços odontológicos dos municípios, 41,7\% o classificaram como ótimo e bom, demonstrando uma maior insatisfação dos usuários quando comparados aos serviços de saúde geral (gráfico 2). Quinze por cento já precisaram de encaminhamento para especialidades odontológicas e, destes, 75,5\% tiveram seus problemas resolvidos. Aqueles que não conseguiram resolver seus problemas alegaram uma demora excessiva e poucas vagas para atendimento, falta de cobertura para determinados procedimentos e deficiência de materiais utilizados no serviço. 


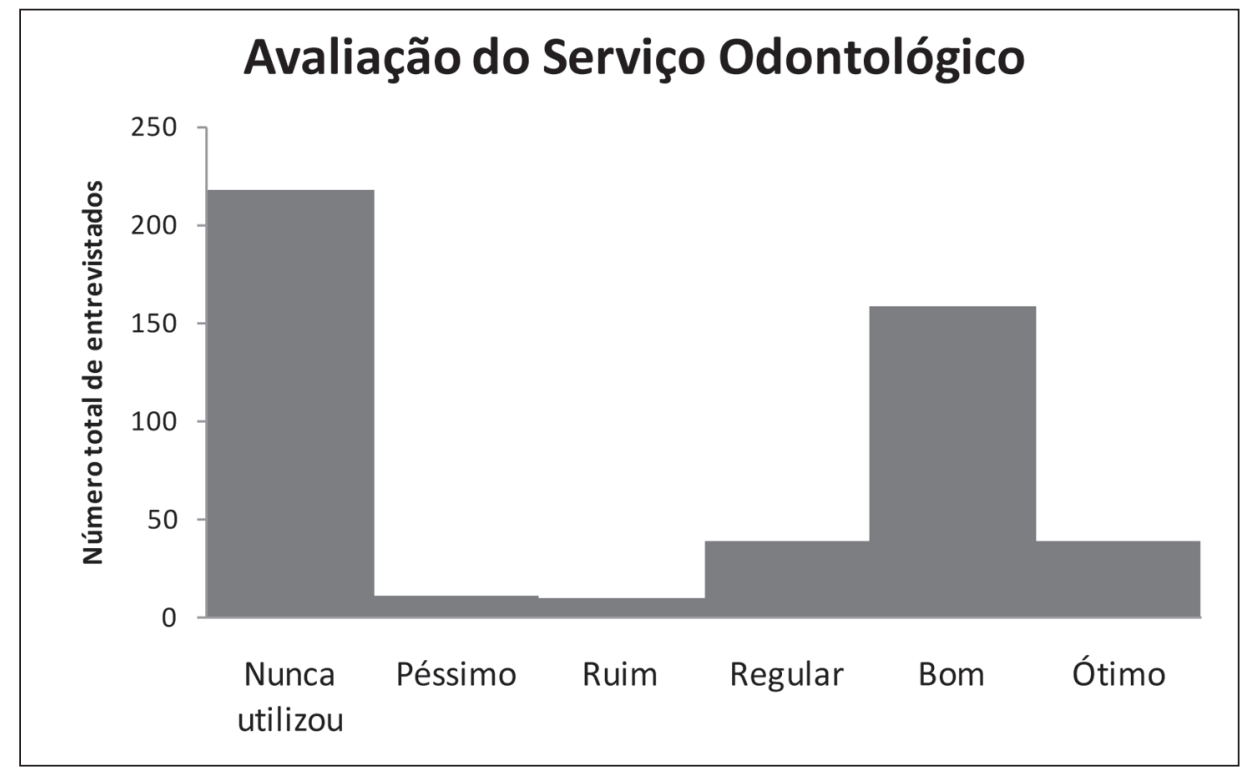

Em um estudo conduzido no país (ANDRADE; FERREIRA, 2006), a necessidade de mais vagas para o atendimento cirúrgico-restaurador e a falta do tratamento reabilitador no serviço odontológico foram as principais queixas relatadas por usuários do sistema público. Em diversas situações, quando as necessidades não são resolvidas no serviço local, não há um esquema de referência / contrarreferência e os problemas ficam sem solução, já que não há como os usuários pagarem por um tratamento, assim como, apesar de nos municípios avaliados em nosso estudo existir um sistema de contrarreferência, representado pela Faculdade de Odontologia de Araçatuba, o serviço não foi capaz de atender toda a demanda, ficando muitos pacientes excluídos do atendimento.

Por fim, foi questionado aos usuários o que poderia ser feito para melhorar os serviços de saúde do município. As respostas foram agrupadas em categorias e listadas as falas mais representativas dos participantes. 


\section{Recursos humanos}

[...] acabar com a demora e fazer os médicos pararem no posto. Falta um bom médico.

[...] mais médicos especialistas pra cada problema.

[...] aumentar o numero de profissionais.

[...] colocar mais médicos; às vezes precisa de médico e não tem, chega com os filhos, é difícil, tem que esperar o atendimento com as crianças [...]

[...] mais dentistas e realização de outros tratamentos.

[...] mais médicos, mais carga horária, pois já cheguei no posto e não tinha médico [...]

Um dos grandes problemas enfrentados pelos municípios de pequeno porte é a falta de recursos humanos. Situação semelhante foi observada em estudo conduzido no Estado de Minas Gerais, segundo o qual as principais deficiências relacionadas à ESF estavam a falta de médicos especialistas na unidade e o número reduzido de profissionais na equipe (RONZANI; SILVA, 2008). É difícil encontrar médicos que residam nos pequenos municípios, sendo que a maioria vem de municípios maiores da região. Essa situação causa uma sensação de falta de proteção ao usuário, que depende do deslocamento do profissional em caso de emergência. Soma-se a isso a remuneração baixa do profissional, o que não estimula novas contratações.

Considerando a demanda por médicos especialistas e a insatisfação com o atendimento, é possível visualizar quão essenciais as ações curativas são na atenção primária. Em análise da trajetória da implantação de serviços de atenção primária no Brasil - especialmente a partir das mudanças no sistema de saúde da década de 1980 -, é possível observar que, ao invés de este campo propiciar a integração da ação curativa com ações de prevenção e promoção da saúde, o que se assiste são a filas de espera por atendimento especializado. Assim parece haver, nesses casos, a importação da lógica dos serviços de emergência e de urgência ao trabalho da atenção primária à saúde (CAMPOS, 2003).

\section{Humanização do atendimento}

[...] acabar com a política e não diferenciar as pessoas.

[...] as agentes deveriam dar mais atenção à população, especialmente a população idosa.

[...] atender com satisfação, alegria [...] 
[...] examinar as pessoas antes de tacar a caneta [...]

[...] pessoal mais preparado, mais humano, ter mais paciência, fazer com amor a profissão.

[...] mais atenção à população [...]

[...] médico mais educado que trate a gente com mais carinho [...]

[...] melhorar o atendimento na recepção, serem mais eficientes, devido a demora, muitas vezes respondem mal pra gente [...]

A humanização do atendimento é uma necessidade gritante nos serviços de saúde. A falta de comprometimento, respeito e atenção por falta do profissional são umas das principais causas de insatisfação do usuário. Deve ser instaurada uma política de acolhimento do usuário, seguindo-se as recomendaçãos da Política Nacional de Humanização do SUS (BRASIL, 2004), que inclua toda a equipe do serviço, desde o guarda ou vigia da unidade até o médico. Apesar de acesso e acolhimento serem elementos de assistência interdependentes, é importante estabelecer suas diferenças para melhor analisar como vêm sendo acolhidos os usuários nos serviços investigados. $\mathrm{O}$ acolhimento não se limita apenas a uma recepção cordial, mas extrapola esse conceito, incluindo a escuta ativa do usuário. Aspectos como vínculo, resolutividade e desempenho profissional estão relacionados com o acolhimento. Também, o atendimento humanizado do paciente proporciona segurança a este e fortifica a relação profissional-paciente, contribuindo inclusive na melhora da saúde do paciente (BRASIL, 2004).

$O$ despreparo da equipe e a falta de um treinamento e capacitação ficam evidentes ao se observar a falta de cuidado no atendimento do paciente. Muitas vezes, o processo de contratação é feito sem concurso público e o favorecimento político para a ocupação dos cargos é marcante, empregando pessoas sem perfil para o trabalho.

\section{Medicamentos}

[...] mais remédio $[\ldots]$

[...] falta remédio, antes tinha, agora quase não tem!

[...] mais remédios que as pessoas usam, pois não tem todos [...]

[...] não faltar remédios, porque as vezes os ricos pegam os remédios e falta para os pobres[...] 
A queixa pela falta de medicamentos deve ser analisada com cautela. Existe uma limitação de recursos financeiros para a compra de remédios nos municípios, o que gera uma cobertura incompleta das necessidades dos usuários. Por outro lado, existe uma tendência cultural do brasileiro pela aquisição de remédios, como se todos os problemas dele só fossem resolvidos por meio destes. A dependência física e emocional pelo medicamento acaba onerando o serviço com uma demanda supervalorizada. Outro problema encontrado advém da população flutuante, que surge à procura de emprego nas temporadas de safra da cana-de-açúcar, já que a fonte econômica principal da região são as usinas de álcool. A população original dos municípios chega a triplicar nos períodos de safra, impossibilitando o serviço de saúde de atender a todos com os recursos disponíveis.

\section{Controle social}

[...] a prefeitura deveria pegar um representante de cada setor da cidade e discutir sobre os problemas da cidade [...]

[...] melhor conscientização do poder público e da população.

[...] participação popular e pressão no prefeito [...]

[...] maior participação popular no conselho [...]

Nem todos os municípios estavam com seus Conselhos Municipais de Saúde em atividade regular. Apesar do direito assegurado pela Constituição (BRASIL, 1988), foram poucos os usuários que se queixaram da falta de controle popular no processo decisório da saúde local. Este tema foi trabalhado posteriormente com a comunidade por meio da discussão e distribuição do Manual do Usuário, buscando maior conscientização da população.

\section{Atendimento organização dos serviços}
[...] melhorar a organização [...]
[...] melhorar o agendamento, porque precisamos de um atendimento mais rápido.
$[\ldots]$ melhorar o atendimento $[\ldots]$
[...] diminuir a fila, dar prioridade a emergências.
[...] melhores condiçôes de atendimento [...]
$[\ldots]$ acabar com o agendamento $[\ldots]$ 
[...] encaminhar mais rápido para atendimento fora da cidade [...]

[...] outros tipos de atendimento odontológico [...]

[...] resolver os problemas na própria cidade.

A principal queixa que se depreende dessas falas é a necessidade do usuário por uma melhor organização do serviço, que proporcione atendimento mais rápido e efetivo. A existência de grandes filas de espera causa um entrave no serviço, torna-o mais lento e pouco resolutivo (OLIVEIRA, 2000). Ao avaliar a acessibilidade aos serviços, Schaedler (2004) propõe a ideia de organização em rede, na qual a população, entre outras coisas, poderia ter acesso irrestrito aos serviços, de modo que quem necessite de atendimento seja dignamente acolhido, independentemente da porta escolhida como meio de acesso.

\section{Prevenção}

[...] conscientização, fazendo uma medicina mais preventiva [...]

[...] mais prevenção [...]

[...] os médicos deveriam dar mais palestras para que todos pudessem participar.

Apesar de algumas falas se referirem à necessidade de atividades de prevenção, estas vêm sendo feitas nos municípios, alguns obtendo melhores resultados que outros, mas todos na tentativa de seguir as recomendações da Estratégia Saúde da Família.

\section{Administração}

$[\ldots]$ bom governo $[\ldots]$

[...] menos burocracia [...]

$[\ldots]$ honestidade por parte dos governantes $[\ldots]$

[...] melhorar a administração e uma união entre a população e o município [...]

$[\ldots]$ união política $[\ldots]$

A burocracia gera dificuldades administrativas que se refletem na qualidade do serviço. A administração pública está sob constante observância e deve procurar meios eficientes para trabalhar, pois o usuário é o primeiro a sentir os seus resultados. 


\title{
Equipamentos / infraestrutura
}

\author{
$[\ldots]$ aparelhos mais novos $[\ldots]$ \\ [...] criação de uma maternidade e mais equipamento, como o de raio-x [...] \\ $[\ldots]$ melhor estrutura $[\ldots]$
}

São necessários maiores investimentos na aquisição de materiais e recursos físicos nos serviços de saúde, pois a maioria deles encontra-se sucateada e em desuso.

Observa-se, a partir destes resultados, a relevância da qualidade do atendimento aos usuários como constructo determinante do seu nível de satisfação, evidenciando a necessidade de o serviço qualificar, cada vez mais, o processo de atendimento ao paciente, enfatizando aspectos tais como agilidade, responsividade e cortesia.

Em estudo de avaliação da qualidade de serviços de saúde (SANTOS, 1995), foi observado que, entre as reclamações dos usuários, aparece com frequência o "não ter conseguido tudo o que precisavam". Entre os itens citados, destacamse a falta da atenção curativa e a dificuldade em conseguir medicamentos. Assim, as principais sugestôes dadas pelos usuários recaíram sobre o aumento de profissionais no quadro e o cumprimento dos horários por parte dos funcionários, principalmente dos médicos. Semelhante situação foi observada em nosso estudo. As falas dos usuários refletem sua percepção individual, e algumas vezes não podem ser generalizadas, como posições políticas partidárias. No entanto, de um modo geral, ficou evidente a deficiência do serviço quanto aos recursos físicos e humanos, carências essas que ganham destaque quando se considera que são municípios de pequeno porte, onde a distância dos grandes centros se torna um agravante considerável.

Pesquisar a respeito da satisfação dos usuários é uma tarefa fundamental para a gestão do serviço prestado, uma vez que seu entendimento pode proporcionar uma avaliação de desempenho sob a perspectiva do usuário, ou cliente, indicando decisões tanto estratégicas quanto operacionais que venham a influenciar no nível de qualidade dos serviços prestados pela organização. $\mathrm{O}$ desenvolvimento de um sistema de avaliação de satisfação pode representar uma importante ferramenta para o desenvolvimento de estratégias de gestão para o setor de serviços. Isso ainda viria a somar-se aos grandes esforços já realizados de padronização e aprimoramento dos serviços da área da saúde, suportados 
pelos inúmeros programas de qualidade que se desenvolvem e são amplamente divulgados em todo o país (MILAN, 2005). A escuta da opinião de quem recebe a assistência é um elemento importante na avaliação dos serviços de saúde, bem como da forma que o sistema de saúde se organiza para viabilizar o atendimento. A incorporação do usuário na avaliação tem sido valorizada não apenas por constituir-se um indicador sensível da qualidade do serviço prestado, mas por estar potencialmente relacionada à maior adequação no uso do serviço.

Os resultados obtidos nesta pesquisa demonstram a insatisfação dos usuários em relação a diversos aspectos do atendimento, como organização dos serviços e da demanda, infraestrutura deficiente, falta de recursos humanos e humanização no atendimento. Apesar de os usuários classificarem os serviços de saúde geral e odontológico como satisfatórios em cerca de metade dos casos, o que se destaca são as queixas inseridas nos relatos dos sujeitos. Na literatura sobre satisfação de usuários, é bastante peculiar observar que, a despeito de variabilidade de formas de se conceber e de medir a satisfação de usuários, a maioria dos estudos, entre abordagens quantitativas e qualitativas, traz como resultado altas taxas de satisfação (ESPEREDIÃO; TRAD, 2005). Este fenômeno é conhecido na literatura como efeito de "elevação" das taxas de satisfação e é reportado mesmo quando as expectativas sobre os serviços são negativas. Era de se esperar que em países em desenvolvimento, como o Brasil, por exemplo, onde o acesso a serviços de saúde de boa qualidade ainda é privilégio de poucos, os usuários manifestassem mais insatisfação com os serviços que lhes são oferecidos.

Quanto às limitações deste estudo, inicialmente deve-se considerar que se trata de uma pesquisa do tipo transversal, apresentando, portanto, as limitações inerentes a esse tipo de pesquisa. Em segundo lugar, os participantes da pesquisa foram os usuários dos serviços de saúde municipal, considerados como todo e qualquer cidadão brasileiro, sendo entrevistados os chefes de família ou respectivos cônjuges. Ao definir o horário comercial para a coleta de dados, o estudo ficou sujeito a uma população enviesada, como se observou em uma amostra predominantemente feminina e de donas de casa. Esta característica da amostra pode ter influencido os resultados, por meio de uma percepção específica como a influência do gênero e saúde, sabidamente importante. No entanto, outras alternativas metodológicas foram testadas e várias tentativas foram feitas para que as entrevistas fossem realizadas com os chefes de família, e não apenas 
com as suas respectivas esposas (já que a maioria dos chefes de família eram homens), incluindo visitas domiciliares durante finais de semana e agendamento prévio, não obtendo sucesso em encontrá-los em casa. Assim, tal deficiência metodológica não pode ser contornada e, apesar do viés de seleção, não invalida os resultados obtidos. Segundo Ferreira Filho et al. (1996), situação semelhante é encontrada na maioria dos estudos realizados em serviços de saúde, nos quais a clientela feminina é sempre predominante.

Por fim, os resultados da pesquisa foram apresentados às equipes de saúde dos municípios envolvidos, a fim de contribuir para a reorientação dos profissionais e para uma possível discussão juntamente com os gestores sobre os problemas relatados pelos usuários. Além disso, houve um esclarecimento sobre a importância da participação popular no SUS, enfatizando-se a ESF como um promotor desta participação e também da importância dos conselhos municipais para discussão, com a comunidade, dos pontos positivos, pontos negativos e alternativas para melhorar o sistema de saúde local.

\section{Conclusão}

O presente estudou apresentou, de uma forma geral, uma avaliação positiva dos serviços de saúde municipais pelos usuários da rede pública, embora tenham sido observadas inúmeras queixas quanto ao atendimento, falta de humanização e acolhimento, longo tempo de espera e filas para o atendimento, deficiência de recursos físicos e materiais. A percepção do usuário é de extrema importância ao se dimensionar o reflexo das ações que vêm sendo desenvolvidas no setor saúde, e serve como vetor de direcionamento e planejamento do serviço.

Concluímos afirmando que o SUS, apesar dos intensos avanços que vem alcançando em seu processo de implementação, ainda tem que promover melhorias na sua organização para que seus princípios sejam efetivos.

\section{Agradecimentos}

Nossos agradecimentos a Fundação de Amparo à Pesquisa do Estado de São Paulo - FAPESP (Processo no 03/07175-9), pelo apoio financeiro cedido a este projeto. 


\section{Referências}

ABEP. Associação Brasileira de Empresas de Pesquisa. Dados com base no Levantamento Sócio Econômico 2000. Disponível em: <http://www.abep.org/codigosguias/ABEP_CCEB_2003. pdf $>$. Acesso em: 23 fev. 2005.

ANDRADE, K.L.C.; FERREIRA, E.F. Avaliação da inserção da odontologia no Programa Saúde da Família de Pompéu (MG): a satisfação do usuário. Ciênc. saúde coletiva, Rio de Janeiro, v. 11, n. 1, p. 123-130, 2006.

BARDIN, L. Análise de conteúdo. 3a ed. São Paulo: Ed. 70, 2004. 223p.

BRASIL. Senado Federal. Constituição da República Federativa do Brasil. Brasília: Senado Federal, 1988.

BRASIL. Ministério da Saúde. Conselho Nacional de Saúde. Resolução no 333/03. Coletânea de Normas para o Controle Social no SUS. Brasília: Ministério da Saúde, 2006.

BRASIL. Ministério da Saúde. Núcleo Técnico da Política Nacional de Humanização. Humaniza SUS. Política Nacional de Humanização: a humanização como eixo norteador das práticas de atenção e gestão em todas as instâncias do SUS. Brasília: Ministério da Saúde, 2004.

CAMPOS, C.E.A. O desafio da integralidade segundo as perspectivas da vigilância da saúde e da saúde da família. Ciênc. saúde coletiva, Rio de Janeiro, v. 8, n. 2, p. 569-584, 2003.

COTTA, R.M.M. et al. Reflexões sobre o conhecimento dos usuários no contexto do Programa de Saúde da Família: a lacuna entre o saber técnico e o popular. Physis: Revista de Saúde Coletiva. Rio de Janeiro, v. 18, n. 4, p. 745-766, 2008.

CREVELIM, M.A. Participação da comunidade na equipe de saúde da família: é possível estabelecer um projeto comum entre trabalhadores e usuários? Ciênc. saúde coletiva. Rio de Janeiro, v. 10, n. 2, p. 323-331, 2005.

ESPERIDIĀO, M.; TRAD, L.A.B. Avaliação da satisfação de usuários. Ciênc. saúde coletiva. Rio de Janeiro, v. 10, supl, p. 303-312, 2005.

FERREIRA FILHO, J.S. et al. Avaliação de qualidade de serviços de saúde mental no Município de Niterói - RJ. A satisfação do usuário como critério avaliador. J Bras Psiquiat., Rio de Janeiro, v. 45, n. 7, p. 393-402, 1996.

FRANCO, S.C.; CAMPOS, G.W.S. Avaliação da qualidade de atendimento ambulatorial em pediatria em um hospital universitário. Cad Saúde Pública., Rio de Janeiro, v. 14, n. 1, p. 61-70, 1998.

GROISMAN, S. et al. A evolução da atenção à saúde no Brasil: o contexto da saúde bucal. Rio de Janeiro: ABOPREV, 2005. 9 p. 
GUEDES, D.G.M.; GARCIA, T.R. Atendimento nos serviços do Sistema Único de Saúde e satisfação do usuário: estudo no município de Campina Grande (PB). Saúde em Debate., Rio de Janeiro, v. 25, n. 59, p. 40-9, 2001.

INSTITUTO BRASILEIRO DE GEOGRAFIA E ESTATÍSTICA. IBGE cidades. Disponível em URL: <http://www.ibge.gov.br/cidadesat/default.php>. Acesso em: 07 abr. 2006. JESUS, P.B.R; CARVALHO, D.V. Percepção de usuários de unidade de saúde da família sobre a assistência à saúde: uma contribuição da enfermagem. REME Rev. min. enferm., Belo Horizonte, v. 6, n. 1-2, p. 48-56, 2002.

KLOETZEL, K. et al. Controle de qualidade em atenção primária a saúde. A satisfação do usuário. Cad Saúde Pública. Rio de Janeiro, v. 14, n. 3, p. 263-268, 1998.

MATEOS, M. et al. Differences in patient satisfaction by gender in users of hospitals in the Cantabrian Health Service. Rev Calid Asist., Barcelona, v. 24, n. 3, p. 104-108, 2009.

MILAN, G.S.; TREZ, G. Pesquisa de satisfação: um modelo para planos de saúde. RAE electron., São Paulo, v. 4, n. 2, dez. 2005. Disponível em: <http://www.scielo.br/scielo. php? script $=$ sci_arttext $\&$ pid $=\$ 1676-56482005000200002 \& \operatorname{lng}=$ pt \&nrm $=$ iso $>$. Acesso em: 01 ago. 2009.

MULLER, A. et al. Um desafio para o cirurgiāo-dentista: a valorização da saúde. UFES Rev. odontol., Vitória, v. 5, n. 2, p. 11-18, 2003.

OLIVEIRA, A.G.R.C. A organização da demanda em serviços públicos de saúde bucal: universalidade, da equidade e da integralidade em saúde bucal coletiva. 2000. 238 p. Tese (Doutorado em Odontologia Preventiva e Social) - Faculdade de Odontologia, Universidade Estadual Paulista, Araçatuba, 2000.

RAMOS, D.D.; LIMA, M.A.D.S. Acesso e acolhimento aos usuários em uma unidade de saúde de Porto Alegre, Rio Grande do Sul, Brasil. Cad S Pública. Rio de Janeiro, v. 19, n. 1, p. 27-34, 2003.

RESENDE, J. et al. A satisfação do usuário com serviços de atendimento odontológico: Faculdade de Odontologia da Universidade do Estado do Rio de Janeiro. Rev. reg. Araçatuba assoc. paul. cir. Dent. Araçatuba, v. 19/20, p. 13-18, 1998/1999.

RICHARDSON, R.J. Pesquisa social: métodos e técnicas. 3a ed. São Paulo: Atlas, 1999. 334 p. RONCALLI, A.G.O. O desenvolvimento das políticas de saúde no Brasil e a consequência do Sistema Único de Saúde. In: PEREIRA, C. A. Odontologia em Saúde Coletiva: planejando ações e promovendo saúde. São Paulo: Artmed, 2003. p. 28-49.

RONZANI, T.M.; SILVA, C.M. O Programa Saúde da Família segundo profissionais de saúde, gestores e usuários. Ciênc. saúde coletiva. Rio de Janeiro, v. 13, n. 1, p. 23-34, 2008. 
SCHNEIDER, H.; PALM, N. Getting to the truth? Researching user views of primary care. Health Policy Plan. London, v. 17, n. 1, p. 32-41, 2002.

SALIBA, N.A. et al. Parceria pela saúde - Projeto desenvolvido no interior do Estado de São Paulo, financiado pela Fapesp, integra instituiçōes educacionais da área da saúde, governo e sociedade civil. Sp.Gov., São Paulo, v. 4, p. 34-37, 2007.

SANTOS, K.T. et al. Agente comunitário de saúde: perfil adequado a realidade do Programa Saúde da Família?. Ciênc. saúde coletiva (online). Rio de Janeiro, 0137/2008. Disponível em: <http://www.abrasco.org.br/cienciaesaudecoletiva/artigos/artigo_int.php?id_ artigo $=2104>$. Acesso em: 02 ago. 2009.

SANTOS, M.P. Avaliação da qualidade dos serviços públicos de atenção à saúde da criança sob a ótica do usuário. Rev. bras. enferm., Brasília, v. 48, n. 2, p. 109-19, 1995.

SCHAEDLER, L.I. Sistema Único de Saúde como rede em prática pedagógica. In: BRASIL. Ministério da Saúde. VER-SUS/Brasil: caderno de textos. Brasília: Editora do Ministério da Saúde, 2004. p. 82-89.

SENNA, M.C.M. Equidade e política de saúde: algumas reflexões sobre o Programa Saúde da Família. Cad S Pública. Rio de Janeiro, v. 18, n. supl., p. 201-211, 2002.

TRAD, L.A.B.; BASTOS, A.C.S. O impacto sociocultural do Programa de Saúde da Família (PSF): uma proposta de avaliação. Cad S Pública. Rio de Janeiro, v. 14, n. 2, p. 429-35, 1998.

WHO. World Health Organization. Oral health surveys: basic methods. 4th ed. Geneva: WHO, 1997. 73 p.

\section{Nota}

${ }^{1}$ Texto inédito. O estudo foi financiado pela Fundação de Amparo à Pesquisa do Estado de São Paulo - FAPESP (Processo no 03/07175-9). Este trabalho recebeu o Prêmio Mário Covas, categoria Gestão de Recursos Humanos, pelo Governo do Estado de São Paulo, em 2007. Não apresenta conflitos de interesse. 


\section{Satisfaction and perception of SUS's users about public health care}

The assessment of the degree of users' satisfaction with the health system is an important indicator to be considered in planning actions. The aim of this study was to evaluate the degree of satisfaction of users of health services and municipal services to be used. This survey was conducted in five municipalities of São Paulo. The sample was selected at random, stratified, and interviewed 471 heads of households or their spouses. Qualitative data were analyzed using content analysis and quantitative data were processed using the statistical software Epi Info. Among the users of the five municipalities, $93.0 \%$ attended the public health care. To $72.0 \%$, health services are solving the problems and needs of the population. Based on access to care, $57.6 \%$ of the population complained about the presence of queues for care. More than half (69.5\%) said they had confidence in the health team, however, many reports demonstrated the lack of a humanized care. In relation to health services, $61.7 \%$ rated them as excellent or good. We conclude that most users are satisfied with the municipal health services, despite the large amount of complaints regarding care, lack of humanization and welcoming, lack of facilities. The user's perception is of utmost importance when scaling the result of actions that have been developed in the health sector, and serves as a vector of direction and planning service.

> Key words: Evaluation in health, Family Health Program, Consumers Satisfaction, Health Services. 